\title{
A wet adhesion inspired biomimetic pad with direction dependence and adaptability
}

\author{
LI MingHe*, HE Bin", QIN HaiYan, ZHOU YanMin, LU HanXiong \& YUE JiGuang \\ Department of Control Science, Tongji University, Shanghai 201804, China
}

Received October 1, 2010; accepted January 5, 2011; published online May 12, 2011

\begin{abstract}
A novel adhesive pad for a climbing robot, inspired by a stick insect's smooth pad, is proposed. The micro structure pattern and inclined fiber of a stick insect's pad are mimicked, and an adhesion force is produced based on its wet adhesion mechanism. Firstly, the wet adhesion model is discussed and the pad is designed; secondly, the adhesion characteristics, adhesion direction dependence and adaptability are analyzed; finally, the proposed pad is fabricated using a thermoplastic elastomer, which is a hydrophilic and viscoelastic material. Experimental results show that the adhesion performance of the pad is good, and normal adhesion and friction can reach to $0.65 \mathrm{~N}$ and $3 \mathrm{~N}$. Furthermore, the adhesion of the pad is direction dependent and self-adaptive.
\end{abstract}

wet adhesion, bionic pad, biomimetic adhesion material, climbing robot, direction dependence of adhesion

Citation: $\quad \mathrm{Li} \mathrm{M} \mathrm{H,} \mathrm{He} \mathrm{B,} \mathrm{Qin} \mathrm{H} \mathrm{Y,} \mathrm{et} \mathrm{al.} \mathrm{A} \mathrm{wet} \mathrm{adhesion} \mathrm{inspired} \mathrm{biomimetic} \mathrm{pad} \mathrm{with} \mathrm{direction} \mathrm{dependence} \mathrm{and} \mathrm{adaptability.} \mathrm{Chinese} \mathrm{Sci} \mathrm{Bull,} \mathrm{2011,} \mathrm{56:}$ 1935-1941, doi: 10.1007/s11434-011-4375-5

Surface adhesion is one of the most critical problems for a climbing robot. In general, vacuum, magnetic, viscous and mechanical interlocking adhesion methods are used as adhesion mechanisms for climbing machines. These adhesion mechanisms have their various advantages. For example, vacuum and viscous adhesion methods are strong while mechanical interlocking adhesion is suitable for rough surfaces [1]. However, disadvantages limit their applications. For example, vacuum adhesion is unavailable in a vacuum environment, viscous adhesion will fail to stick to the surface after initial success and magnetic adhesion can only be used on specific metal surfaces. However, geckos, crickets, ants and beetles have strong climbing ability regardless of the roughness of the contact surface. Their adhesive pads have evolved over millions of years. Consequently the design of an adhesive pad for a climbing robot based on insect techniques has considerable merit.

In recent times, wet adhesion mechanisms of animals have received much scientific attention, and many important outcomes have been achieved. Preliminary results show that

*Corresponding authors (email: hebin0910@hotmail.com; limingheliming@sina.com) the dry adhesion force comes mainly from van der Waals force, whereas the wet adhesion force comes mainly from surface tension or a capillary force [2-4]. At the same time, biomimetic adhesive pads with good adhesion and direction dependence have been developed based on etching, mechanical microscopic fabrication or carbon nanotube growth technologies [5-11]. However, most of these biomimetic pads were fabricated using a material with a high elastic modulus, and they generally require considerable pressure (or preloading) to achieve effective contact. The adhesion is not self-adaptive to the load and the pad is not self-cleaning, so their applications are limited.

So far, almost all the biomimetic adhesive pads proposed have been based on dry adhesion mechanisms, inspired by the gecko setae. Wet adhesion insects (e.g. the stick insect) have soft and smooth pads which can mold to the shape of a surface, and are able stick to different kinds of smooth and rough surfaces. However, little research has been reported on this type of biomimetic material. A wet adhesion pad, fabricated using silicone, was proposed in our previous work and applied to the newly developed wheel-leg climbing robot [12]. This wet adhesion mechanism proved to be 
effective for the wall climbing robot. However, a weakness in the pad and lack of direction dependence limited the application, and large preloading or pressure was needed to produce effective adhesion. This paper proposes a novel wet adhesion inspired biomimetic pad with direction dependence and adaptability, and its properties are studied both theoretically and experimentally.

\section{Wet adhesion mechanism}

Pads (Figure 1(a),(b)) of insects, like ants and stick insects, can generate adhesion forces several times their own weight $[13,14]$. The smooth pad secretions form a thin layer of liquid film between the pad and contact surface (the thickness of the film is smaller than several microns) which can produce adhesion forces when this type of insect climbs up walls, as shown in Figure 1(c) and (d) [4,15]. Most researchers believe the wet adhesion force comes from surface tension and capillary forces rather than viscous forces [15].

(i) Adhesion model. The wet adhesion model proposed by Federle et al. is shown in Figure 2 [4]. A liquid film (or liquid bridge) is formed between the pad and the contact surface to produce the wet adhesion force. According to the model, the liquid volume is constant, so when the pad is pulled or squeezed, the liquid bridge will shrink or expand rapidly. Supposing that $R$ is the radius of the pad, and $\theta_{1}$ and $\theta_{2}$ are the contact angles. For the adhesion force which comes from surface tension and capillary forces, the total normal adhesion force (vertical to the contact surface) $F_{\text {wet }}$ can be written as [16]

$$
F_{\text {wet }}=-\pi r_{\text {wet }}^{2} \Delta p+2 \pi r_{\text {wet }} \gamma \cos \theta_{1},
$$

where $r_{\text {wet }}$ is the radius of contact area (i.e. effective contact area), $\gamma$ is the coefficient of surface tension of secretion, and $\Delta P$ is the pressure difference between the inside and the outside of the secretion.

(ii) Friction model. For the model shown in Figure 2, we assume that the pad is sliding on the surface. Static friction coming from viscous force should be very small; while the dynamic friction $\left(F_{\mathrm{fr}}\right)$ comes from the shear stress, which can be written as [4]

$$
F_{\text {fr }}=\frac{\pi r^{2}}{h} v \eta,
$$

where $v$ is the moving velocity of the pad, $h$ is the height of the thin secretion bridge, and $\eta$ is the viscosity of the secretion which is dependent on the properties of secretion and $h$. When $h<10 \mathrm{~nm}$, the friction will be very large.

Eqs. (1) and (2) and results from [4,15,17], show that normal adhesion and friction are proportional to the contact area, and inversely proportional to the thickness of the secretion film. Additionally, the friction is proportional to the shear rate of secretion. This gives the basic principles for the design of a biomimetic pad.

\section{Pad design}

For improving adhesion performance, most biomimetic pads are fabricated by a material with a high elastic modulus such as carbon nanotubes, which generally require a large pressure (or preloading) to achieve effective contact. This makes it difficult to stick to rough surfaces and limits their applications. However, a wet adhesion based biomimetic pad can be fabricated using a low young's modulus, and a highly viscoelastic material, and the pad only requires a thin liquid film with a thickness of several microns to obtain good adhesive performance. In addition, the fabrication method is much simpler than that for biomimetic setae.

The nronosed biomimetic pad is based on the stick insect. It has the same appearance as the stick insect's smooth pad, which contains a semi-elliptic contact surface and inclined fibers (Figure 3).

(i) The semi-elliptic surface of the pad consists of three parts: the far end, bottom and near end (Figure 3(b)). The far end is $1.5-4 \mathrm{~mm}$ thick and stiff, and is used for supporting the main preloading produced by the robot, while the pad is in contact with a surface (such as a vertical wall). The shear stress here is high. The bottom is thin $(1.5 \mathrm{~mm})$ and soft, so it can make good contact with the rough surface. When approaching a surface, the bottom is the first part of the pad that comes into contact. The near end is also very thin $(1.5 \mathrm{~mm})$ and soft, it may or may not touch the surface depending on the preloading.

(ii) There are special micro-structures (patterns) distributed on the surface of the insect's pad (Figure 1(b)), which can help with secretion and increase the adhesion forces $[16,17]$. Thus, a special square pattern is designed for the proposed pad (Figure 4). The sides of the square are 200 $\mu \mathrm{m}$, and the width and depth of the grooves between
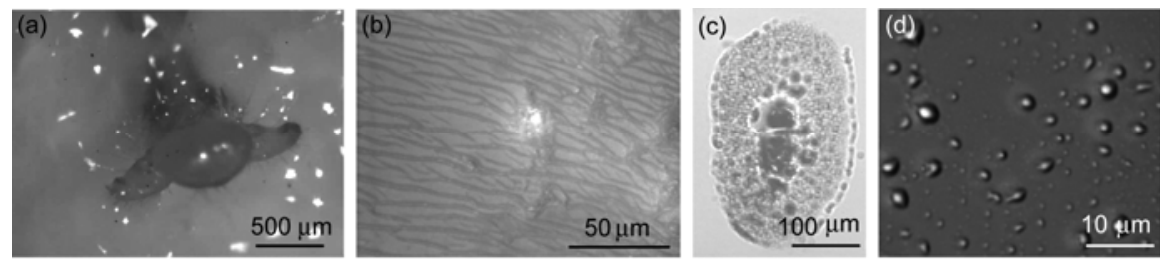

Figure 1 Smooth pad and secretion of insects. (a) Smooth pad; (b) pattern of the pad; (c) footprint of pad; (d) secretion. 


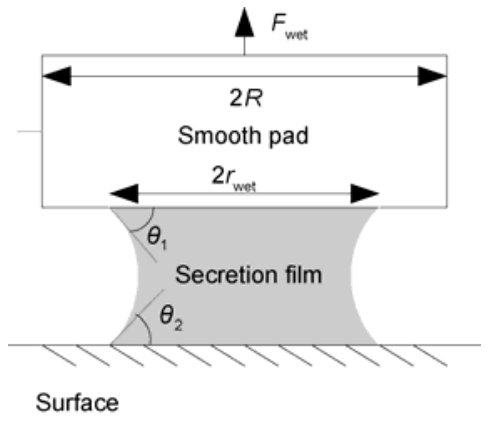

Figure 2 Liquid bridge model of wet adhesion [4].

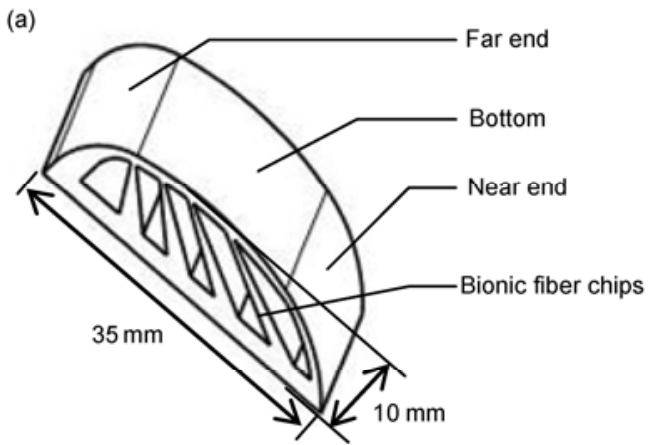

(b)

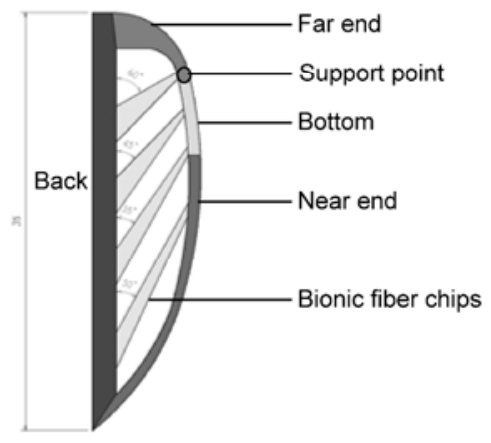

Figure 3 Biomimetic pad. (a) 3D view of the pad; (b) lateral view of the pad.

squares are 100 and $300 \mu \mathrm{m}$, respectively (Figure 4(b)). According to the experimental results and the adhesive tape peeling theory [16], the best adhesion performance is obtained when the squares are placed at an oblique angle of $45^{\circ}$.

(iii) Inside the smooth pad of an animal there are branched topological micro-structures [18], and those close to the surface are very thin, which make the pad softer and easier to achieve contact with the surface. However, the branched structures are too complex to mimic in biomimetic pads using the current technology. Thus, the fiber design of the pad is simplified in this paper: fiber chips are adopted instead of branched fibers, and those far from the contact surface or near the back are $3 \mathrm{~mm}$ thick, and close to the contact surface are $1.5 \mathrm{~mm}$ thick.

(iv) The fibers are inclined at $30^{\circ}-60^{\circ}$ (Figure $3(\mathrm{~b})$ ) and the fiber chips all point from the body of the robot to the rear. The closer to the rear, the stiffer the fiber chip, and the greater the friction. The closer to the body, the softer the fiber chip and the more flexible the pad, the greater the normal adhesion. Moreover, the inclined fiber chips mean the adhesion of the pad depends on direction, so the robot can control the adhesion effectively. When the pad slides towards the robot's body, adhesion will increase rapidly, and when it slides away, adhesion will decrease rapidly.

\section{Theoretical analysis of the pad}

\subsection{Contribution of the surface pattern to adhesion}

(i) Positive effects of the surface pattern on normal adhesion. The proposed pad can be simplified as a disk (which is in full contact with the surface) where $r_{\text {wet }}$ is the radius and $h$ is the thickness of the thin liquid film. For a pad without a patterned surface, the normal adhesion force $\left(F_{\text {wet }}\right)$ can be calculated by eq. (1); for a pad with a patterned surface (Figure 4), the whole contact area is sub-divided into $n$ contact blocks, each with a radius of $r_{\text {wet }} / \sqrt{n}$. Thus, the total normal adhesion force $\left(F_{\mathrm{a}}\right)$ of the contact area can be calculated by eq. (3):

$$
\begin{aligned}
F_{\mathrm{a}} & =n\left(-\pi\left(\frac{r_{\mathrm{wet}}}{\sqrt{n}}\right)^{2} \Delta p+2 \pi\left(\frac{r_{\mathrm{wet}}}{\sqrt{n}}\right) \gamma \cos \theta_{1}\right) \\
& =-\pi r_{\text {wet }}{ }^{2} \Delta P+2 \sqrt{n} \pi r_{\text {wet }} \gamma \cos \theta_{1},
\end{aligned}
$$

where $F_{\mathrm{a}}$ is much larger than $F_{\text {wet }}$ in eq. (3). We found that the capillary force component does not change compared with eq. (1); however, the surface tension component is $\sqrt{n}$ times larger.

(ii) Positive effects of the surface pattern on parallel adhesion. Parts of the pad make contact with the wall directly and produce friction component $f$. The pattern on the surface helps increase the friction force (Figure 5). In this case the total parallel adhesion $\left(F_{\mathrm{p}}\right)$ can be calculated by eq. (4):

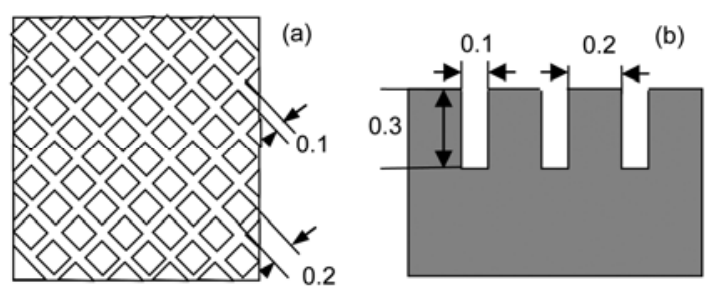

Figure 4 Design of the surface of the pad. (a) Pattern of the surface (mm); (b) lateral view of the pattern of the pad $(\mathrm{mm})$.

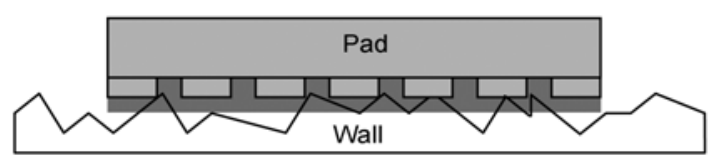

Figure 5 Pad with pattern in contact with the wall. 


$$
F_{\mathrm{p}}=f+F_{\mathrm{rc}}=\mu N+\frac{\pi r^{2} v \eta}{h}
$$

here, $\mu$ is friction coefficient, $N$ is the preload of the pad, and $F_{\text {rc }}$ is the shear force of the liquid film.

(iii) Positive effects of the surface pattern on secretion. Efficient adhesion can only happen when the thickness of the thin secretion film between the pad and the wall is less than several microns. The secretion procedure should finish in a very short time if the insect or robot tries to climb fast, but the secretion process becomes progressively more difficult as the secretion film becomes thinner. We consider two parallel pans which are immersed in a liquid with viscosity $\eta_{2}$, where $R_{\text {pan }}$ is the radius, and $F$ is the pressure force between the two pans. The expected time $\left(t_{\mathrm{R}}\right)$ for the distance between the two pans to decrease from $h_{2}$ to $h_{1}$ [19] can be calculated as

$$
t_{R}=\frac{3 \pi}{4} \cdot \frac{\eta_{2} R_{\mathrm{pan}}^{4}}{F} \cdot\left(\frac{1}{h_{1}^{2}}-\frac{1}{h_{2}^{2}}\right) .
$$

According to eq. (5), $t_{\mathrm{R}}$ becomes longer as the secretion film becomes thinner. However, the secretion process would be easier if there was a pattern on the surface of the pan. We assume the contact area of the pan is subdivided into $n$ contact blocks, and $r_{\text {pan }}$ is the radius of each contact block. When $r_{\text {pan }}<R_{\text {pan }} / \sqrt{n}$, the expected secretion time would be $n$ times shorter than for the pad without a pattern.

\subsection{Simulation of the direction dependence of a biomimetic pad}

Direction dependence occurs if the adhesion forces are different when sliding the pad in different directions, which is a very important factor for the ability of insects or animals to stick and unstick to surfaces. There have been some biomimetic setae with direction dependence reported; however, there have been no reports on a smooth biomimetic pad based on wet adhesion. The inclined fiber chips can make the proposed biomimetic pad exhibit the direction dependence of adhesion.

To verify the direction dependence of the pad, ANSYS finite element analysis software, was used to simulate the deformation and the dynamics while the pad is sliding on the contact surface. The solid element, No.92, was used in the simulation. The material's elastic modulus is $50 \mathrm{kPa}$, and its Poisson's Ratio is 0.33 . The deformation results of the simulation are illustrated in Figures 6 and 7.

The initial conditions are $50 \mathrm{mN}$ of preload vertical to the pad and $200 \mathrm{mN}$ of pulling force pointing towards the robot body (Figure 6). The deformation and the distribution of stress are illustrated in Figure 6(a) and 6(b) respectively. The results show that the angles between the fiber chips and contact surface decrease and the contact area of the near end increases when pulling the pad towards the robot body, so the adhesion forces will increase accordingly. We can see from Figure 6(b) that the highest stress happens at the supporting point of the pad, which means that the pad would not easily be peeled off from this point, and the friction here is the highest. We also find that the largest deformation happens at the near end of the pad; however the stress here is quite small. Consequently, this part is very soft and a small preload would lead to efficient contact with the surface.

An initial condition of $200 \mathrm{mN}$ of pushing force pointing to the far end is shown in Figure 7. The deformation and the distribution of stress are illustrated in Figure 7(a) and 7(b) respectively. The deformation results show that the angle between the fiber chips and contact surface is augmented and the contact area of the near end decreases rapidly when pushing the pad away, so the adhesion will also decrease rapidly. We also find that the largest deformation happens at the near end, however, the stress here is quite small. Consequently, this part is very soft, so the pad can be very easily peeled off (Figure 7(a)). Figure 7(b) shows that the stress of the supporting point is also very high during the peeling off process.

In conclusion, the pad exhibits the direction dependence of adhesion.
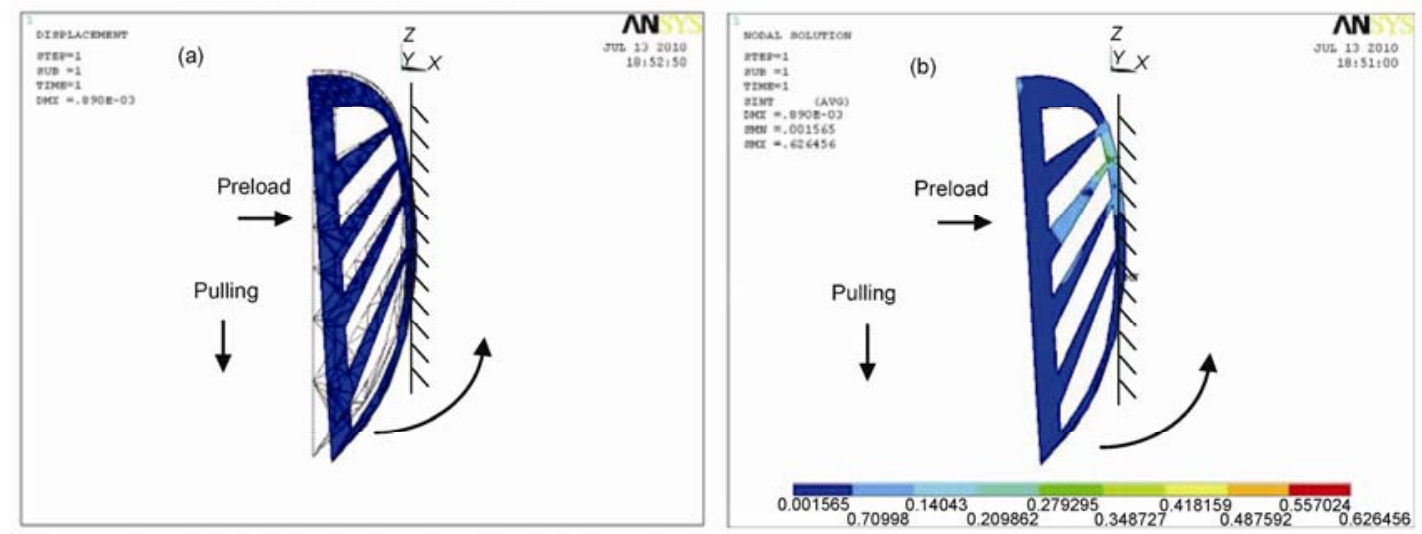

Figure 6 Deformation (a) and dynamics (b) of the pad when pulling on the surface. 

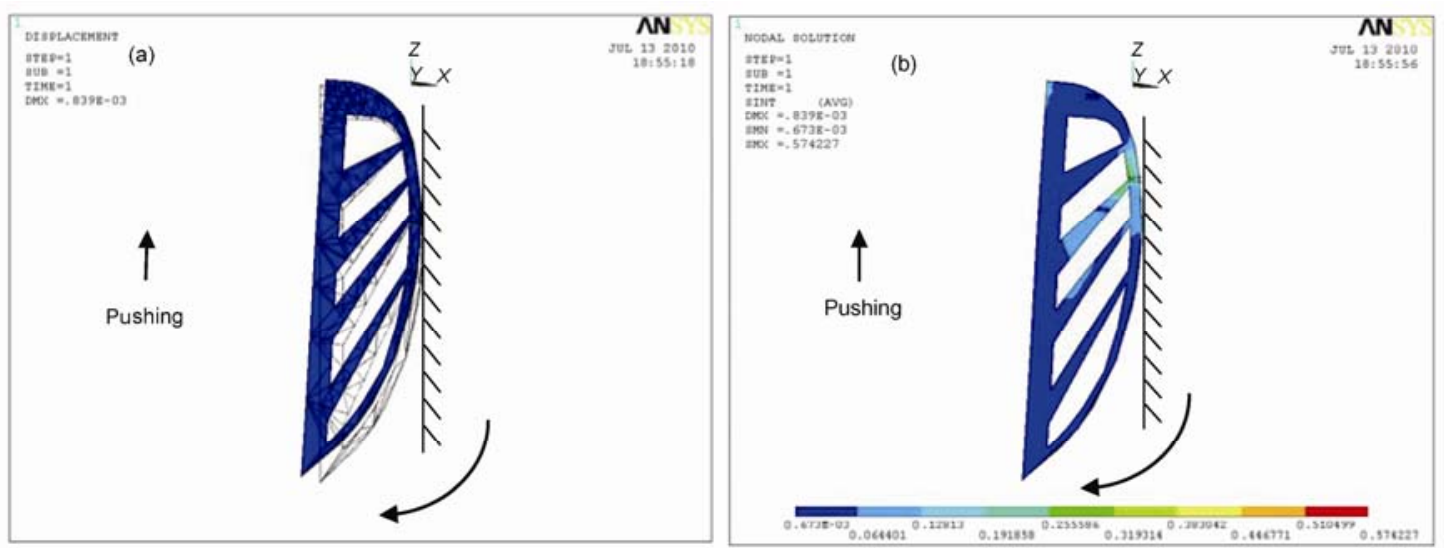

Figure 7 Deformation (a) and dynamics (b) of the pad when pushing on the contact surface.

\subsection{Self-adaptability mechanism of adhesion}

The adaptability not only means that the soft pad can adhere to a vertical surface with varied roughness, but also that the adhesion will increase or decrease due to different loads (parallel to the contact surface) on the robot. If the pad does not peel off after the load increases, then the bionic fiber chips will be bent. The pad is modeled as an elastomer, whose spring constant is $K$, where the preload (vertical to the contact surface) is proportional to the load (parallel to the contact surface) when the increased load is small, and the scale is $p$ (which is a constant here). Then the decreased thickness $(\Delta x)$ of the pad due to the increased load can be calculated as

$$
\Delta x=\frac{p W_{\mathrm{a}}}{K},
$$

where $W_{\mathrm{a}}$ is the increased load. For a climbing robot, the decreased thickness of the pad equals the decreased distance between the climbing surface and the center of gravity of the robot [12]. Thus, the normal adhesion needed by the robot will not change as fast as the load, which means that the pad is self-adaptive to the load.

The near end of the pad can be simplified as a plane (Figure 3(b)). Here, $b$ is the width, and the angle between the near end and the back of the pad is $\alpha$. Then the contact area $(S)$ can be calculated as

$$
S=\frac{b \Delta x}{\sin \alpha}+S_{0}
$$

where $S$ and $S_{0}$ refer to the current contact area, and the initial contact area (before the fiber chips bent (prior to loading)), respectively.

Our results and those of other researchers show that the wet adhesion force $\left(F_{\text {wet }}\right)$ is proportional to the increased effective contact area. Suppose $\mu$ is the proportional coefficient between them, then

$$
F_{\text {wet }}=\mu S=\frac{\mu b \Delta x}{\sin \alpha}+\mu S_{0} .
$$

Considering eq. (6), eq. (8) can be written as

$$
F_{\text {wet }}=\frac{\mu b p W_{\mathrm{a}}}{K \sin \alpha}+\mu S_{0} .
$$

As is shown in eq. (9), $F_{\text {wet }}$ will increase adaptively with increasing $W_{\mathrm{a}}$. However, the relationship between $K$ and $W_{\mathrm{a}}$ is a nonlinear coupling. Therefore, the relationship between $F_{\text {wet }}$ and $W_{\mathrm{a}}$ is also nonlinear. To simplify the model, $K$ can be considered the mean value of each fiber chip's spring constant $\left(K_{i}\right)$, which is

$$
K=\sum_{i=1}^{N} K_{i} / n .
$$

Here $n$ is the total number of fiber chips. $K_{i}$ can be obtained in a similar way to the calculation of the spring constant of the setae in [11].

In conclusion, the distance between the center of gravity of the robot and wall surface will decrease, and the contact area of the pad will increase with increasing load, hence the pad is self-adaptive to the load.

\section{Experimental results and analysis}

\subsection{Methods}

The biomimetic pad was fabricated from a thermoplastic elastomer (TPE, CHAOLONG Plastics Co., LTD, Dong Guan City) using a mold casting method. The TPE's melting point was $180^{\circ}-220^{\circ}$, the Young's modulus was about 40-60 kPa and the hardness was between 0 and 20. The material is thermoplastic, viscoelastic and hydrophilic, and is easily damped in a non-sealed environment. The properties of the TPE are similar to those of the insect's pad. The pad shown in Figure 8, is $10 \mathrm{~mm}$ high, $24 \mathrm{~mm}$ wide and 36 $\mathrm{mm}$ long. The area of the bottom surface is about $240 \mathrm{~mm}^{2}$.

To validate the performance of the proposed pad, the adhesion/friction forces of the biomimetic pad were tested with different preloads, with/without a thin liquid (water) 


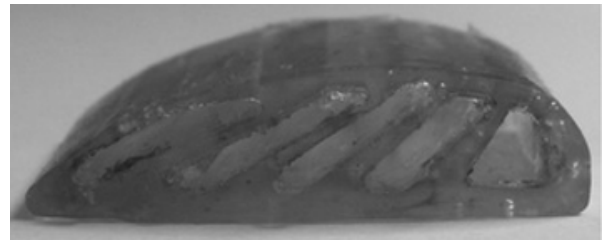

Figure 8 Biomimetic pad made of TPE.

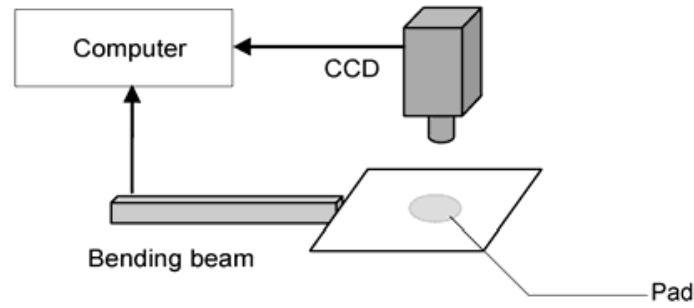

Figure 9 Adhesion force measurement device.

film and with different sliding directions. The measurement device is shown in Figure 9 and consists of a micro-force sensor (NS-YB04D, Shanghai TM Automation Instruments Co. Ltd, Shanghai) which is bending beam-based, a CCD camera (A602f, Basler Vision Technologies), a computer and other components. The maximum resolution of the micro-force sensor is $1 \mathrm{mN}$. The resolution of CCD is $800 \times$ 600. The speed of data acquisition is $100 \mathrm{f} / \mathrm{s}$. When measuring the friction, we placed the pad fully in contact with the sensor's glass and slide the pad on the glass at a speed of $0.01 \mathrm{~m} / \mathrm{s}$. The sensor collected the micro-force data, while the CCD recorded the contact area in real time. When measuring the normal adhesion force, the pad approached the glass vertically at a velocity of $0.01 \mathrm{~m} / \mathrm{s}$, while the adhesion force and contact area data were collected in real time.

\subsection{Results and analysis}

(i) Experiments for verifying the wet adhesion mechanism. As the TPE is a high hydrophilic material, with $50 \%$ or less relative humidity, it resulted initially in droplets covering the surface, so it was difficult to compare between dry and wet adhesion of the TPE. Here we simply measured and compared the adhesion when sliding the pad on the glass surface both with and without a thin water film wiped on the surface.

The adhesion performance of the pad has been shown to be low when the water film is thick [12], thus after wiping the water on the surface, we waited for about 2 minutes to make sure the water film was very thin (about $10 \mu \mathrm{m}$ thick), before we started to measure the adhesion and friction forces. Here, the sample size was 10 (10 pads), we tried 10 times for each one, and took the mean value as the final result (Figure 10). The results show that:

(1) The adhesion and friction performances were good

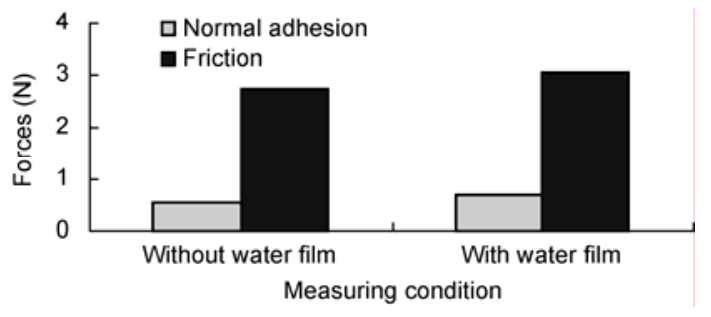

Figure 10 Wet adhesion forces of the pad.

both with and without a water film wiped on the glass surface, and the friction forces were larger than the normal adhesion forces. This was due to the good hydrophilic and viscoelastic properties of the TPE and design of the pad.

(2) A thin secretion film between the pad and contact surface improved the adhesion performance. However, the thickness of the water film was hard to control, thus the adhesion forces of the pad were not as good as in nature. With a thin water film on the glass, the normal adhesion and friction of the pad reached $2.8 \mathrm{mN} / \mathrm{mm}^{2}$ and $12.7 \mathrm{mN} / \mathrm{mm}^{2}$ respectively. However, the normal adhesion and friction of a stick insect can reach up to $36 \mathrm{mN} / \mathrm{mm}^{2}$ and $132 \mathrm{mN} / \mathrm{mm}^{2}$.

(ii) Relationship between adhesion and preload. With $50 \%$ relative humidity and without a liquid film between the pad and the contact surface, we measured the adhesion force with different preloads. For each preload, we tried 10 times on one pad, and took the mean value as the final result. The results (Figure 11) show that irrespective of the normal adhesion or friction, the adhesion force increased with increasing preloading. When we calculated the contact area from the video, we found that the change of adhesion due to the change of the contact area kept a linear relationship. In Section 3.3, we argued that the preload would increase with the increase in the robot load while the pad was touching the vertical surface, thus the results indicate that the pad is self-adaptable.

(iii) Direction dependence test of the pad. With the same initial conditions as the previous tests, when pulling or pushing the pad with the same preload, the adhesion measured results are shown in Figure 12. Here, the sample size was 10 (10 pads), we tried 10 times for each one, and took the mean value as the final result. The results show that, irrespective of the normal adhesion or friction, the adhesion was much greater when pulling the pad on the glass surface. In particular for normal adhesion, we achieved about $0.55 \mathrm{~N}$ when pulling, and $-30 \mathrm{mN}$ when pushing, which demonstrated clear direction dependence for adhesion. We calculated the contact area from the video and found that the difference in the adhesion forces mainly came from the differences in the contact areas, while maintaining a linear relationship.

\section{Conclusions}

After introducing a wet adhesion mechanism, a smooth wet 

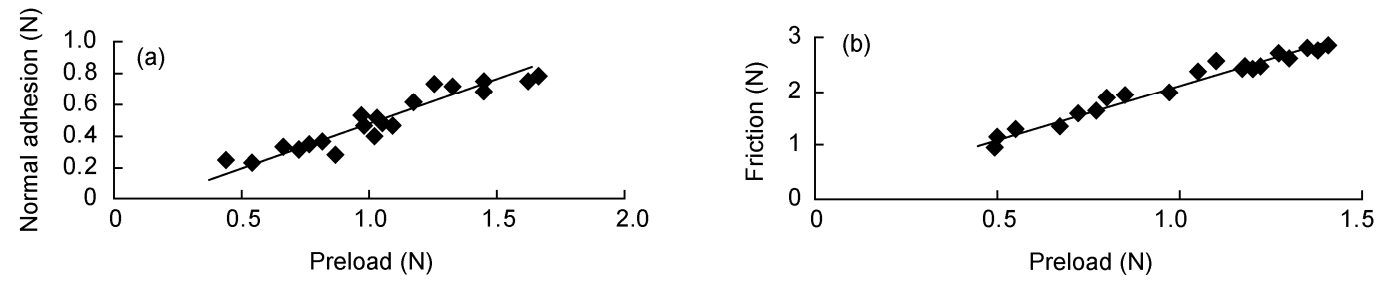

Figure 11 Relationship between preloading and adhesion. (a) Relationship between adhesion and preloading; (b) relationship between friction and preloading.

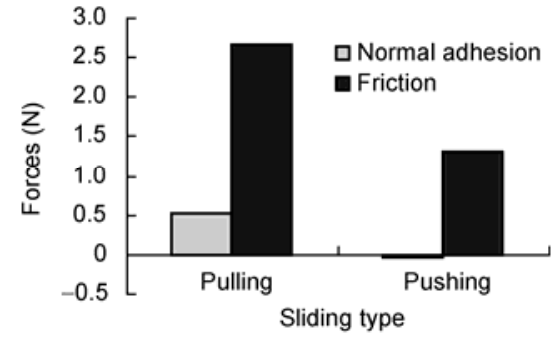

Figure 12 A comparison of adhesion when pulling and pushing on the glass surface.

adhesion based biomimetic pad for a climbing robot was proposed in this paper. Special square patterns on the surface helped improve wet adhesion performance, and inclined fiber chips inside the pad made the adhesion of the pad direction dependent and self-adaptive. Experimental results showed that the wet adhesion performance of the pad was good, with normal adhesion and friction reaching 0.65 and $3 \mathrm{~N}$ respectively when sliding on wet glass surfaces. Furthermore the direction dependence was very clear. In future work, we will test the adhesion performances on rough surfaces.

This work was supported by the National Natural Science Foundation of China $(50405045,61040056)$ and the National High-Tech Research \& Development Program of China (2007AA04Z253). Also thanks to Walter Federle's research group's help on the work.

1 Daltorio K A, Horchler A D, Gorb S, et al. A small wall-walking robot with compliant adhesive feet. In: IEEE/RSJ International Conference on Intelligent Robots and Systems, 2005 August 2-6, Edmonton, Alberta, Canada. IEEE Press, 2005. 4018-4023

2 Autumn K, Liang Y A, Hsieh S T, et al. Adhesive force of a single gecko foot-hair. Nature, 2000, 405: 681-684

3 Gorb S N. Uncovering insect stickiness: Structure and properties of hairy attachment devices. Am Entomol, 2005, 51: 31-35

4 Federle W, Riehle M, Curtis A S G, et al. An integrative study of insect adhesion: Mechanics and wet adhesion of pretarsal pads in ants. Integr Comp Biol, 2002, 42: 1100-1106
5 Zhang H, Guo D J, Dai Z D. Progress on gecko-inspired micro/ nano-adhesion arrays. Chinese Sci Bull, 2010, 55: 409-417

6 Qu L T, Dai L M, Morley S, et al. Carbon nanotube arrays with strong shear binding-on and easy normal lifting-off. Science, 2008, 322: $238-242$

7 Aksak B, Murphy M P, Sitti M. Gecko inspired micro-fibrillar adhesives for wall climbing robots on micro/nanoscale rough surfaces. In: 2008 IEEE International Conference on Robotics and Automation, 2008 May 19-23, Pasadena, California, USA. IEEE Press, 2008. 28862890

8 Gorb S N, Varenberg M, Peressadko A, et al. Biomimetic mushroom-shaped fibrillar adhesive microstructure. J R Soc Interface, 2006, 4: $271-275$

9 Lee J, Bush B, Maboudian R, et al. Gecko-inspired combined lamellar and nanofibrillar array for adhesion on nonplanar surface. Langmuir, 2009, 25: 12449-12453

10 Wang $\mathrm{H} \mathrm{J}$, Mei T, Wang X H. Adhesion array design of a novel biomimetic gecko crawling robot (in Chinese). Robot, 2006, 28: 191194

11 Liu B, Zhang H, Guo D J, et al. Design, fabrication and adhesion test of lean bio-inspired seta (in Chinese). Tribology, 2009, 29: 393-398

$12 \mathrm{Li} \mathrm{M} \mathrm{H}, \mathrm{He} \mathrm{B}$, Yue J G, et al. Wet adhesion mechanism and the application on wall climbing robot (in Chinese). Robot, 2010, 32: 577585

13 Drechsler P, Federle W. Biomechanics of smooth adhesive pads in insects: Influence of tarsal secretion on attachment performance. J Comp Physiol A, 2006, 192: 1213-1222

14 Federle W, Rohrseitz K, Holldobler B. Attachment forces of ants measured with a centrifuge: Better 'wax-runners' have a poorer attachment to a smooth surface. J Exp Biol, 2000, 203: 505-512

15 Zhou Q, He B, Yue J G. Source of attachment forces of ants and the secretion effect (in Chinese). Chin J Theor Appl Mech, 2007, 39: 428432

16 Zhou Q. Research of driving theory and mechanics modeling for biomimetic wet-adhesion micro-robots (in Chinese). Dissertation for the Dcotoral Degree. Shanghai: Tongji University, 2009

17 Persson B N J. Wet adhesion with application to tree frog adhesive toe pads and tires. J Phys Condens Matter, 2007, 19: 1-16

18 Clemente C J, Federle W. Pushing versus pulling: Division of labor between tarsal attachment pads in cockroaches. Proc R Soc B, 2008, 275: $1329-1336$

19 Reynolds $\mathrm{O}$. On the theory of lubrication and its application to $\mathrm{Mr}$ Beauchamp Tower's experiments, including all experimental determination of the viscosity of olive oil. Phil Trans R Soe Lond, 1886, $157-234$

Open Access This article is distributed under the terms of the Creative Commons Attribution License which permits any use, distribution, and reproduction in any medium, provided the original author(s) and source are credited. 